\title{
Coexistence of low and high overlap phases in a supercooled liquid: an integral equation investigation
}

\author{
Jean-Marc Bomont, ${ }^{1}$ Giorgio Pastore, ${ }^{2}$ and Jean-Pierre Hansen ${ }^{3}$ \\ 1) Université de Lorraine, LCP-A2MC, EA 3469, 1 Bd. François Arago, \\ Metz F-57078, France $e^{\text {a) }}$ \\ 2) Università di Trieste, Dipartimento di Fisica, strada Costiera 11, \\ 34151 Grignano (Trieste), Italy ${ }^{\mathrm{b})}$ \\ 3) Université Pierre et Marie Curie, UMR 8234 PHENIX, Paris, \\ France and \\ Department of Chemistry, University of Cambridge, Cambridge CB2 1EW, \\ $U K^{\mathrm{c})}$
}

(Dated: 24 February 2017)

The pair structure, free energy and configurational overlap order parameter Q of an annealed system of two weakly coupled replicas of a supercooled "soft sphere" fluid are determined by solving the hypernetted-chain (HNC) and self-consistent RogersYoung (RY) integral equations over a wide range of thermodynamic conditions $\rho$ (number-density), $\mathrm{T}$ (temperature) and inter-replicas couplings $\varepsilon_{12}$. Analysis of the resulting effective (or Landau) potential $\mathrm{W}(\rho, \mathrm{T} ; \mathrm{Q})$ and of its derivative w.r.t. $\mathrm{Q}$ confirms the existence of a "precursor transition" between weak and strong overlap phases below a critical temperature $T_{c}$ well above the temperature $T_{o}$ of the "ideal glass" transition observed in the limit $\varepsilon_{12} \rightarrow 0$. The precursor transition is signalled by a loss of convexity of the potential $\mathrm{W}(\mathrm{Q})$ and by a concomitant discontinuity of the order parameter $\mathrm{Q}$ just below $\mathrm{T}_{c}$, which crosses over to a mean-field-like van der Waals loop at lower temperatures. The HNC and RY equations lead to the same phase transition scenario, with quantitative differences in the predicted temperatures $\mathrm{T}_{c}$ and $T_{o}$.

PACS numbers: 64.70.Q-, 61.20.Gy, 05.20.Jj

Keywords: Liquid-glass transition, Random first order transition

\footnotetext{
a)Electronic mail: jean-marc.bomont@univ-lorraine.fr

b)Electronic mail: pastore@ts.infn.it

c)Electronic mail: jph32@cam.ac.uk
} 


\section{INTRODUCTION}

When a liquid is quenched below its freezing point, it undergoes a number of successive transformations associated with a gradual loss of ergodicity and a concomitant dramatic slowing down of the microscopic dynamics. Below a so-called "kinetic glass-transition" at a temperature $T_{d}$, ergodicity is broken within a mode-coupling approximation, but eventually restored via activated jump processes ${ }^{1}$. The local dynamics tends to become heterogeneous, characterized by mesoscopic, dynamically active regions coexisting with quiescent regions over increasingly long periods of time ${ }^{2,3}$. Upon further cooling (or compression), the Maxwell relaxation time increases dramatically and eventually becomes comparable to experimental time scales (say $10^{3} \mathrm{~s}$ ). At this point the system falls out of equilibrium on such time scales, and thermodynamic properties (like the molar volume or the enthalpy under constant pressure conditions) experience an abrupt change of slope at a cooling-rate dependent "glasstransition" temperature $T_{g}{ }^{4}$. The underlying free energy landscape is characterized by an exponentially (with system size) large number of local minima, separated by high barriers ${ }^{5}$. The supercooled liquid remains trapped in one of these metastable states for increasingly long periods of time and the broken ergodicity entails characteristic glassy behavior, i.e. the supercooled system behaves like a disordered (amorphous) solid. Mean-field spin-glassinspired theories point to the existence of an "ideal" glass transition (or "random first-order transition", RFOT) at a Kauzmann temperature $T_{K}{ }^{6}$ where the configurational entropy of the infinitely slowly cooled system vanishes ${ }^{7}$. In view of the infinite equilibration time required to reach the ideal glass state, the transition is not directly observable experimentally, and even less by computer simulations.

More recently, it has been suggested that the RFOT might be preceded by a "precursor transition" occurring at higher temperatures, signaling the coexistence of two phases corresponding to low and high values of the mean overlap Q between configurations of a supercooled system and a frozen configuration of the same system ${ }^{8-10}$. The low $\mathrm{Q}$ phase is associated with the supercooled liquid, whose atoms are still capable of exploring parts of the configuration space far removed from the frozen reference configuration, while the high Q phase corresponds to a glass phase where the atoms remain localized close to atoms of the frozen reference configuration, which acts like a disordered external field. Such a situation is reminiscent of that of a fluid trapped in a porous medium ${ }^{11}$, and will be referred to as 
the "quenched case". An alternative situation, which is more readily accessible to computer simulations and integral equation theories, is that of two weakly coupled identical replicas of the system. Atoms of opposite replicas attract each other via a short-ranged pair interaction, thus forming long-lived pairs ("molecules") at sufficiently low temperatures, such that the configurational overlap of the two replicas is large, as in a glass; this situation is referred to as the "annealed case"12,13. In both quenched and annealed cases, the "precursor transition" terminates at a critical temperature $\mathrm{T}_{c}$, but the criticality is characterized by different critical exponents which coincide with their Ising values only in the "annealed" case according to a finite-size scaling analysis ${ }^{12,13}$.

In the present paper we extend our earlier integral equation work on the ideal glass transition to investigate the "precursor transition" in the annealed case. Our earlier work was based on a "pedestrian" model of two weakly-coupled replicas of a system of "soft spheres" ${ }^{14,15}$ or of Lennard-Jones ${ }^{16}$ atoms. The pair structure, configurational overlap and the free energy of this binary "mixture" were calculated from careful numerical solutions of the hypernetted-chain (HNC) integral equation ${ }^{17}$ and of thermodynamically self-consistent integral equations ${ }^{18,19}$ over a wide range of thermodynamic conditions and inter-replica couplings $\varepsilon_{12}$. Taking the $\varepsilon_{12} \rightarrow 0$ limit, these calculations pointed to the existence of a supercooled liquid (L) phase, an "ideal" glass phase $\mathrm{G}_{2}$, and of a previously undetected glass branch of solutions $\mathrm{G}_{1}$ of higher free energy over a range of temperatures. A first order phase transition between $\mathrm{L}$ and $\mathrm{G}_{2}$ phases was found to occur at a density-dependent temperature $\mathrm{T}_{o}(\rho)$, as determined by the intersection of the free energies of the two phases. The transition is characterized by a discontinuous jump of the order parameter Q (as in the related work by Mézard and Parisi ${ }^{20}$ ), but also by a weak discontinuity (about 1\%) of the molar volume $v=1 / \rho$. $\mathrm{T}_{o}$ was found to be comparable to independent, approximate estimates of the Kauzmann temperature $\mathrm{T}_{K}{ }^{14}$.

In the following Sections we focus on the widely used "soft sphere" model. Exploiting our earlier free-energy data, we construct the effective (or Landau) potential $W(Q)$ as a function of the overlap order parameter $Q$, and the thermodynamic variables $\rho$ and $T$ via a Legendre transformation to switch from the variables $\left(\rho, T, \varepsilon_{12}\right)$ to the variables $(\rho, T, Q)^{8-10,12,13}$.

The effective potential $W(Q)$, as well as the static susceptibility $\chi(Q)$ (proportional to the mean square fluctuation of Q) will then allow us to map out the coexistence curve and the critical point of the "precursor transition". 


\section{THE TWO-REPLICAS MODEL}

We consider a binary mixture of two weakly coupled replicas (labeled 1 and 2) of a "soft sphere" fluid, i.e. of a system of $\mathrm{N}$ atoms in a volume $\mathrm{V}$ (number density $\rho=N / V$ ) interacting via the purely repulsive pair potential:

$$
v_{11}(r)=v_{22}(r)=v(r)=\varepsilon\left(\frac{\sigma}{r}\right)^{12}=\frac{\varepsilon}{x^{12}}
$$

where $\varepsilon$ and $\sigma$ determine the energy and length scales, and $x=r / \sigma$. The reduced dimensionless temperature and density are defined as $T^{*}=k_{B} T / \varepsilon$ and $\rho^{*}=\rho \sigma^{3}=1 / v^{*}(v$ being the volume per particle in each replica). The reduced, excess thermodynamic properties depend on a single dimensionless variable $\Gamma=\rho^{*} /\left(T^{*}\right)^{\frac{1}{4}}$. Atoms of opposite replicas interact via the short-ranged, attractive pair potential:

$$
v_{12}(x)=-\varepsilon_{12} w(x)
$$

For $w(x)$ we have adopted the simple form ${ }^{20}$

$$
w(x)=\left[\frac{c^{2}}{x^{2}+c^{2}}\right]^{6} ;
$$

the dimensionless range parameter $c$ is chosen such that the range of the attraction is significantly shorter than $\sigma$, to ensure that one atom of replica 1 can interact with at most one atom of replica 2 due to the strong repulsion (1) between atoms of the same replica; in practice $c<1$. Denoting a configuration of replica $\alpha$ by $\mathbf{R}^{\alpha}=\left(\mathbf{x}_{1}^{\alpha}, \ldots, \mathbf{x}_{\mathbf{N}}^{\alpha}\right) ; \alpha=1,2$, the total potential energy of the two-replicas system reads:

$$
V_{N, N}\left(\mathbf{R}^{1}, \mathbf{R}^{2}\right)=V_{N}\left(\mathbf{R}^{1}\right)+V_{N}\left(\mathbf{R}^{2}\right)-\varepsilon_{12} \Phi_{N, N}\left(\mathbf{R}^{1}, \mathbf{R}^{2}\right)
$$

where:

$$
\begin{gathered}
V_{N}\left(\mathbf{R}^{\alpha}\right)=\sum_{i} \sum_{j>i} v\left(\left|\mathbf{x}_{i}^{\alpha}-\mathbf{x}_{j}^{\alpha}\right|\right) \\
\Phi_{N, N}\left(\mathbf{R}^{1}, \mathbf{R}^{2}\right)=\sum_{i} \sum_{j} w\left(\left|\mathbf{x}_{i}^{1}-\mathbf{x}_{j}^{2}\right|\right) \\
\equiv N q_{1,2}\left(\mathbf{R}^{1}, \mathbf{R}^{2}\right)
\end{gathered}
$$

$q_{1,2}$ is the overlap function ${ }^{8}$. The mean overlap (or order parameter) is defined as

$$
Q\left(\rho, T ; \varepsilon_{12}\right)=\left\langle q_{1,2}\right\rangle=4 \pi \rho^{*} \int_{0}^{\infty} g_{12}(x) w(x) x^{2} d x
$$


where $g_{12}(x)$ is the inter-replica pair distribution function of the binary "mixture"; the intrareplica p.d.f. will be denoted by $g(x)=g_{11}(x)=g_{22}(x)$. The angular brackets in eqn. (5) denote a statistical average with the Boltzmann weight $\exp \left\{-\beta V_{N, N}\left(\mathbf{R}^{1}, \mathbf{R}^{2}\right)\right\} / Z_{N, N}$, where $\beta=1 / k_{B} T$ and $Z_{N, N}$ is the configuration integral

$$
Z_{N, N}\left(V, T ; \varepsilon_{12}\right)=\int d \mathbf{R}^{1} \int d \mathbf{R}^{2} \exp \left\{-\beta\left[V_{N}\left(\mathbf{R}^{1}\right)+V_{N}\left(\mathbf{R}^{2}\right)-\varepsilon_{12} \Phi_{N, N}\left(\mathbf{R}^{1}, \mathbf{R}^{2}\right)\right]\right\}
$$

Note that $q_{1,2}=1$ in the case of perfect overlap $\left(\mathbf{R}^{1} \equiv \mathbf{R}^{2}\right)$, and hence $Q \leq 1$. When the two replicas are completely decoupled $\left(\varepsilon_{12}=0\right), g_{12}=1$ for all $x$, and $Q$ reduces to its "random overlap" value $Q_{r}=7 \pi^{2} \rho^{*} c^{3} / 128$, as calculated from eqs. 3 and 5 .

Starting from an initial state where the two replicas are weakly coupled $\left(\varepsilon_{12}>0\right)$, the supercooled liquid (L) and Glass (G) branches may be distinguished by different values of the order parameter $Q$ in the limit $\varepsilon_{12} \rightarrow 0^{14,15,20}$. In the L phase

$$
\lim _{\varepsilon_{12} \rightarrow 0} Q\left(\rho, T ; \varepsilon_{12}\right)=Q_{r}
$$

while in the ideal glass state, the positions $\left\{\mathbf{x}_{i}^{1}\right\}$ and $\left\{\mathbf{x}_{j}^{2}\right\}$ of the atoms of the two replicas remain localized close to disordered equilibrium positions $\left\{\mathbf{X}_{i}\right\}$; the resulting strong interreplicas correlations hence imply that:

$$
\lim _{\varepsilon_{12} \rightarrow 0} Q\left(\rho, T ; \varepsilon_{12}\right) \gg Q_{r}
$$

The RFOT is hence expected to be characterized by a discontinuous jump of the order parameter $Q$ in the thermodynamic limit ${ }^{8}$.

In our earlier work ${ }^{14,15}$, the HNC and the thermodynamically self-consistent RogersYoung $(\mathrm{RY})^{18}$ integral equations for the pair structure of the two-replicas system were solved numerically over a wide range of thermodynamic states $\Gamma$ and $\varepsilon_{12}$ (for a brief reminder, see Appendix A). Three branches of solutions $\left(L, G_{1}\right.$ and $\left.G_{2}\right)$ were mapped out and the resulting free energies and order parameters point to an alternative RFOT scenario involving both a strong discontinuity of $Q$ and a weak discontinuity of the volume per particle $v^{*}$ at the ideal glass transition. In order to gain access to the conjectured precursor transition we now switch from a free energy analysis to an effective potential representation via a Legendre transformation ${ }^{8-10,13}$. 


\section{EFFECTIVE POTENTIAL AND OVERLAP FLUCTUATIONS}

The excess Helmholtz free energy per atom is defined by:

$$
f\left(\rho, T ; \varepsilon_{12}\right)=\frac{F^{e x}\left(\rho, T ; \varepsilon_{12}\right)}{2 N}=-\frac{k_{B} T}{2 N} \log \left[\frac{Z_{N, N}\left(V, T ; \varepsilon_{12}\right)}{V^{2 N}}\right]
$$

Remembering eqn.(6), it is easily verified that

$$
Q\left(\rho, T ; \varepsilon_{12}\right)=\left\langle q_{1,2}\left(\mathbf{R}^{1}, \mathbf{R}^{2}\right)\right\rangle_{\varepsilon_{12}}=-2\left(\frac{\partial f\left(\rho, T ; \varepsilon_{12}\right)}{\partial \varepsilon_{12}}\right)_{\rho, T}
$$

Notice the factor 2 , due to the definition of $f\left(\rho, T ; \varepsilon_{12}\right)$ as free energy per particle of the system of two coupled replicas. A Legendre transformation from the variables $\left(\rho, T ; \varepsilon_{12}\right)$ to the variables $(\rho, T ; Q)$ defines a new thermodynamic potential, usually referred to as the effective (or Landau) potential $W$ :

$$
W(\rho, T ; Q)=2 f\left(\rho, T ; \varepsilon_{12}(Q)\right)+Q \varepsilon_{12}(Q)=\min _{\varepsilon_{12}}\left[2 f\left(\rho, T ; \varepsilon_{12}\right)+\varepsilon_{12} Q\right]
$$

provided $Q=\left\langle q_{1,2}\left(\mathbf{R}^{1}, \mathbf{R}^{2}\right)\right\rangle_{\varepsilon_{12}}$.

Reciprocally:

$$
2 f\left(\rho, T ; \varepsilon_{12}\right)=\min _{Q}\left[W\left(\rho, T ; \varepsilon_{12}\right)-\varepsilon_{12} Q\right]
$$

which implies that

$$
W^{\prime}(\rho, T ; Q)=\left(\frac{\partial W(\rho, T ; Q)}{\partial Q}\right)_{\rho, T}=\varepsilon_{12}
$$

The effective potential $W(Q)$ may be associated with a constrained Boltzmann distribution ${ }^{9,12}$

$W(\rho, T ; Q)=-\frac{k_{B} T}{N} \log \left[\frac{1}{V^{2 N}} \int d \mathbf{R}^{1} \int d \mathbf{R}^{2} \exp \left\{-\beta\left[V_{N}\left(\mathbf{R}^{1}\right)+V_{N}\left(\mathbf{R}^{2}\right)\right]\right\} \delta\left(q_{1,2}\left(\mathbf{R}^{1}, \mathbf{R}^{2}\right)-Q\right)\right]$

which determines the overlap distribution function:

$$
P_{0}(Q)=\left\langle\delta\left[q_{1,2}\left(\mathbf{R}^{1}, \mathbf{R}^{2}\right)-Q\right]\right\rangle_{0} \sim \exp \{-\beta N W(\rho, T ; Q)\}
$$

The subscript 0 signifies that the inter-replicas coupling $\varepsilon_{12}$ is set to zero in eqns. (4a) and (6).

Returning to the definition (9) of $Q\left(\rho, T ; \varepsilon_{12}\right)$ and remembering eqns. (4a, 4b) and (6), one may easily check that: 


$$
\left(\frac{\partial Q}{\partial \varepsilon_{12}}\right)_{\rho, T}=-\left(\frac{\partial^{2} 2 f}{\partial \varepsilon_{12}^{2}}\right)_{\rho, T}=\beta\left\langle\left[q_{1,2}\left(\mathbf{R}^{1}, \mathbf{R}^{2}\right)-\left\langle q_{1,2}\left(\mathbf{R}^{1}, \mathbf{R}^{2}\right)\right\rangle_{\varepsilon_{12}}\right]^{2}\right\rangle_{\varepsilon_{12}} \equiv \frac{1}{N k_{B} T} \chi\left(\rho, T ; \varepsilon_{12}\right)
$$

where the static susceptibility $\chi$ is, as usual, determined by the mean square fluctuation of the microscopic order parameter. Phase coexistence between low Q and high Q states is associated with the non-convexity of the effective potential W(Q) below a critical temperature $T_{c}$. In practice, for a given thermodynamic state $(\rho, T)$ the free energy per particle $f\left(\rho, T ; \varepsilon_{12}\right)$ and the order parameter $Q$ are calculated as functions of $\varepsilon_{12}$. The Legendre transformation (10) is then used to determine the effective potential $W(\rho, T ; Q)$. Plotting $W(\rho, T ; Q)$ as function of $T$ along a given isochore will signal non-convexity and hence phase separation below $T_{c}$. The results obtained for the soft sphere model using the HNC or RY integral equations are presented in the following section. In view of eqn. (12), $W(Q)$ (for fixed $\rho, T$ ) may also be calculated by thermodynamic integration:

$$
W\left(Q_{a}\right)-W\left(Q_{b}\right)=\int_{Q_{b}}^{Q_{a}} W^{\prime}(Q) d Q=\int_{Q_{b}}^{Q_{a}} \varepsilon_{12}(Q) d Q
$$

\section{MAPPING OUT THE PHASE DIAGRAM}

The results presented throughout this Section are for a reduced density $\rho^{*}=\rho \sigma^{3}=1$, so that $\Gamma=\left(T^{*}\right)^{-\frac{1}{4}}$. All energies (including $\varepsilon_{12}$ ) are expressed in units of the soft-sphere coupling $\varepsilon$. It should be kept in mind that in the limit of vanishing inter-replicas coupling $\varepsilon_{12}$, the reduced excess thermodynamic properties, e.g. $f^{*}=f / k_{B} T$, and properly scaled pair distribution functions (cf. eqn. (4) of ref. ${ }^{14}$ ) are functions of $\Gamma$ only.

\section{A. HNC results}

The RFOT is expected to occur at $\Gamma \simeq 1.65^{14,15}$. The "precursor transition" must hence occur at higher temperatures, i.e. lower values of $\Gamma$. Results in the interval $1.25<\Gamma<1.35$ and for a range parameter $c=0.3$ in the inter-replica potential $w(x)$ (eqn (3)) are presented in Figs. 1-3. Along each isotherm the variation of the order parameter $Q$ with $\varepsilon_{12}$ is monitored, using a very small increment $\Delta \varepsilon_{12}=0.005$, as shown in Fig. 1. For $\varepsilon_{12}=0$, the random overlap value $Q_{r}=0.0146$ is recovered; $Q$ increases more and more rapidly 


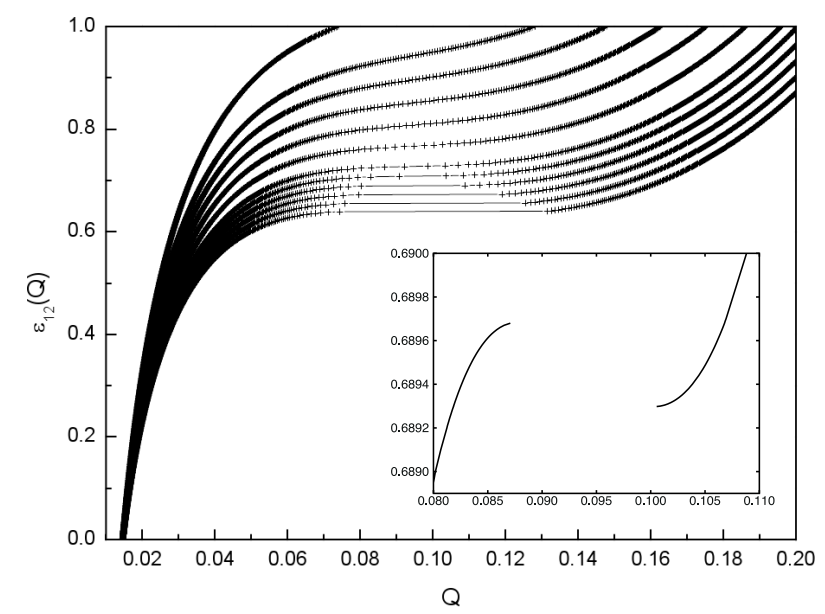

FIG. 1. HNC results for the order parameter $Q$ versus inter-replicas coupling $\varepsilon_{12}$ "isotherms" for couplings $\Gamma=1.25,1.27,1.28,1.29,1.30,1.31,1.32,1.325,1.33,1.335,1.34,1.345$ (from top to bottom) and for range parameter $c / \sigma=0.3$. A discontinuous jump of $\mathrm{Q}$ is observed for $\Gamma \gtrsim 1.33$. The inset shows a strongly magnified version of the jump at Gamma $=1.33$.

with $\varepsilon_{12}$ as $\Gamma$ increases. At $\Gamma=1.33$, a clear-cut discontinuity occurs at $\varepsilon_{12}=0.69$, where $Q$ jumps from $Q=0.0802$ to $Q=0.109$, before increasing further. Such a jump of the $\mathrm{Q}$ values is due to the appearance of a region where the coupled HNC integral equations admit no solutions. The discontinuity $\Delta Q$ increases with increasing coupling $\Gamma$ (i.e. as the temperature is lowered). In the inset of Fig. 1, an enlargement of the curve for $\Gamma=1.33$ is shown. Such a behavior is typical for all the curves up to 1.40. Thus, the curves $\varepsilon_{12}(Q)$ are reminiscent of the mechanically stable part of the mean field isotherms $P(\rho)$ near and below the liquid-gas critical temperature $T_{c}$ below which the fluid separates into a lowdensity vapor and a high-density liquid phase. However, at variance with the mean field case, in this range of couplings, the HNC equations do not have thermodynamically unstable solutions. The latter appear at higher couplings as discussed below. In the present case, low $Q$ and high $Q$ states coexist below the precursor transition critical point characterized by a zero slope inflection point of the $\varepsilon_{12}(Q)$ curve; the corresponding $\Gamma_{c}$ lies between $\Gamma=1.32$ and $\Gamma=1.33$. Some of the resulting $W(\Gamma ; Q)$ vs. $Q$ curves are plotted in Fig. 2 . The effective potential function is seen to be convex for $\Gamma \leq 1.32$; convexity is lost for larger $\Gamma$. A Maxwell common-tangent construction leads to values of the order parameter $Q$ of the coexisting states (listed in Table I) comparable to those estimated from the $\varepsilon_{12}(Q)$ curve; the discrepancy reflects the thermodynamic inconsistency of HNC equation. 


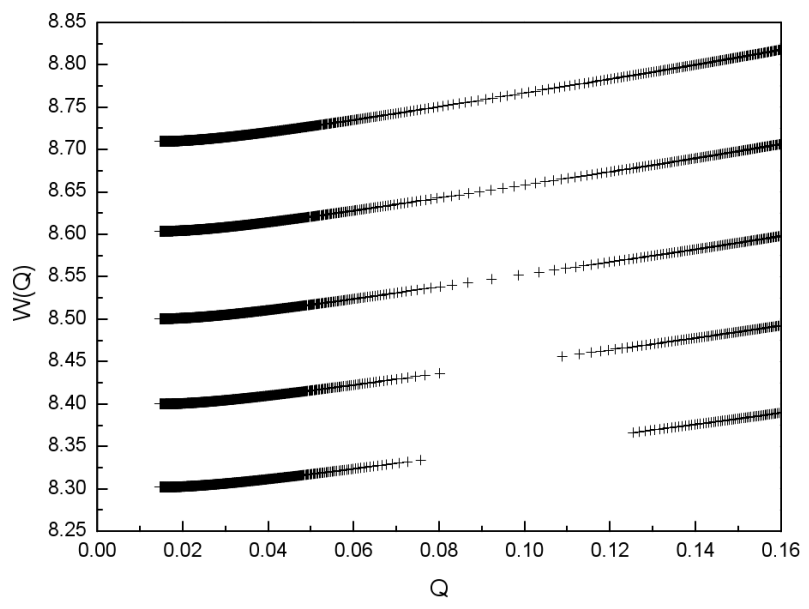

FIG. 2. Effective potential $\mathrm{W}(\mathrm{Q})$ (in units of $\varepsilon$ ) versus order parameter $\mathrm{Q}$ for $\Gamma=1.30,1.31,1.32$, 1.33 and 1.34 (from top to bottom) as calculated from $\mathrm{HNC}$ and $c / \sigma=0.3$. For $\Gamma \gtrsim 1.33, \mathrm{~W}(\mathrm{Q})$ is no longer a convex function of $\mathrm{Q}$.

TABLE I. Values of $T^{*}, Q_{L}, Q_{G_{2}}, \varepsilon_{12}$ and $s_{\text {conf }}$ for several values of $\Gamma$ and $\mathrm{c}=0.3$ and 0.5 within the HNC approximation.

\begin{tabular}{ccccccc}
\hline \hline$\Gamma$ & $T^{*}$ & $\mathrm{c}$ & $Q_{L}$ & $Q_{G_{2}}$ & $\varepsilon_{12}$ & $s_{\text {conf }}$ \\
\hline 1.33 & 0.3196 & 0.3 & 0.0802 & 0.109 & 0.69 & 0.062 \\
1.34 & 0.3102 & 0.3 & 0.0758 & 0.125 & 0.66 & 0.11 \\
1.40 & 0.2603 & 0.3 & 0.0442 & 0.162 & 0.46 & 0.21 \\
1.45 & 0.2262 & 0.3 & 0.0334 & 0.186 & 0.33 & 0.22 \\
& & 0.5 & 0.1480 & 0.286 & 0.23 & 0.14 \\
1.60 & 0.1526 & 0.3 & 0.0171 & 0.248 & 0.054 & 0.082 \\
& & 0.5 & 0.0766 & 0.393 & 0.039 & 0.079 \\
1.63 & 0.1447 & 0.3 & 0.0152 & 0.260 & 0.013 & 0.023 \\
& & 0.5 & 0.0698 & 0.408 & 0.010 & 0.023 \\
1.64 & 0.1382 & 0.3 & 0.0146 & 0.264 & 0.001 & 0.0018 \\
& & 0.5 & 0.0675 & 0.413 & 0.0005 & 0.0018 \\
\hline \hline
\end{tabular}

The mean square fluctuation of the microscopic order parameter determines the static susceptibility $\chi$ (cf. eqn. (15)). As $\Gamma$ increases, $\chi\left(\Gamma ; \varepsilon_{12}\right)$ sharpens, and its amplitude increases rapidly, but does not diverge at criticality, a well-known deficiency of the HNC 


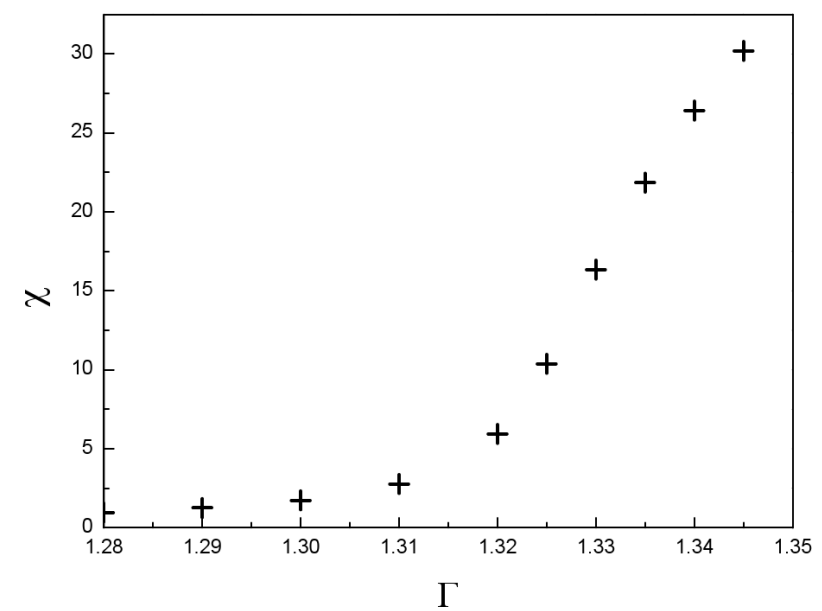

FIG. 3. Amplitude of the dimensionless susceptibility $\chi\left(\rho, T ; \varepsilon_{12}\right)$, corresponding to the maximum of $\chi$ as a function of $\varepsilon_{12}$, versus $\Gamma$ (HNC and $c / \sigma=0.3$ ).

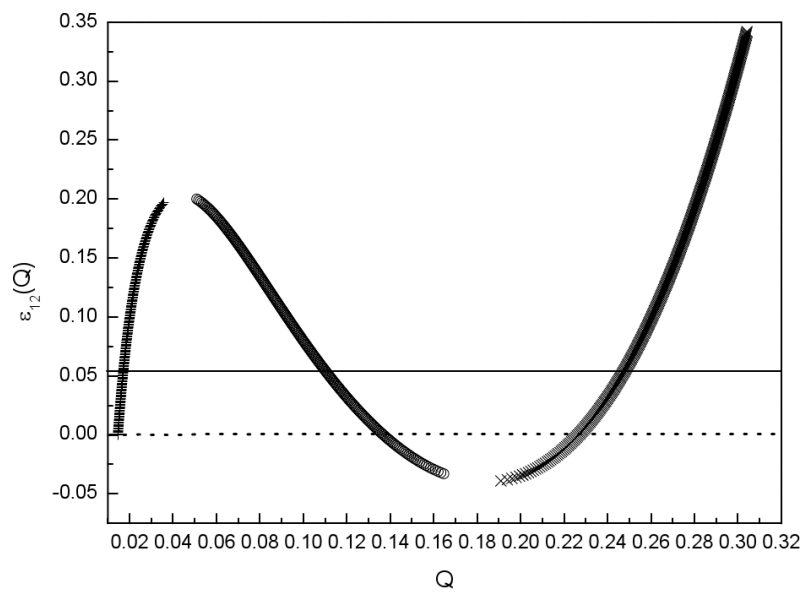

FIG. 4. Same as Fig. 1, but for $\Gamma=1.6 . \varepsilon_{12}(\mathrm{Q})$ exhibits a marked van der Waals-like loop, with two small discontinuities of $Q$ between $L$ (pluses) and $G_{1}$ (circles), and between $G_{1}$ and $G_{2}$ (crosses) branches. The continuous horizontal line corresponds to the Maxwell construction.

equation, as illustrated in Fig. 3, where the maximum of $\chi\left(\Gamma ; \varepsilon_{12}\right)$ as a function of $\varepsilon_{12}$ is plotted over a range of $\Gamma$ values.

The scenario illustrated in Figs. 1-3 changes drastically upon approaching the ideal glass transition. The HNC results for $\varepsilon_{12}(Q)$ as a function of $Q$ are shown in Fig. 4, for $\Gamma=1.6$. Above $\Gamma=1.4$, a new branch of solutions appears, leading to the $\mathrm{L}, \mathrm{G}_{1}$ and $\mathrm{G}_{2}$ states predicted in our earlier work ${ }^{14,15}$. Along the $\mathrm{L}$ branch, $Q$ increases with $\varepsilon_{12}$, up to $\varepsilon_{12} \simeq 0.21$, where $Q$ undergoes a small discontinuous jump towards the $\mathrm{G}_{1}$ branch, along 


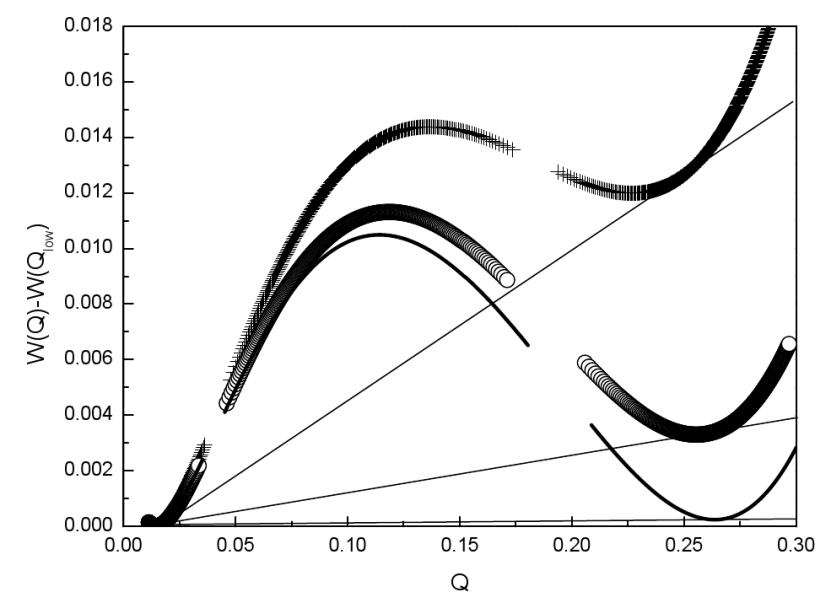

FIG. 5. The effective potential $\mathrm{W}(\mathrm{Q})$ minus its value for the low $\mathrm{Q}(\mathrm{L})$ phase as a function of $\mathrm{Q}$ for $\Gamma=1.6$ (pluses), 1.63 (circles), and 1.64 (line) (HNC and $c / \sigma=0.3)$. The streight lines for the Maxwell common-tangent constructions are also shown.

which $Q$ decreases as $\varepsilon_{12}$ increases. A second discontinuity of $Q$ is observed at a slightly negative value of $\varepsilon_{12}$ towards the $\mathrm{G}_{2}$ branch, along which $Q$ increases again with $\varepsilon_{12}$. Overall the $\varepsilon_{12}(Q)$ curves in the strong-coupling region are reminiscent of van der Waals loops in mean-field theory of liquid-vapor coexistence. However, similarly to the case of the liquidgas phase coexistence in one-component systems ${ }^{21,22}$, HNC theory definitely shows a more complex behavior with the presence of narrow intervals where no solution can be found. The loop implies multi-valued solutions for $-0.04<\varepsilon_{12}<0.205$.

The corresponding effective potential calculated from eqn. (10) is plotted in Fig. 5. It exhibits a low $Q$ minimum associated with the $\mathrm{L}$ branch, a high $Q$ minimum associated to the $\mathrm{G}_{2}$ branch; the two minima are separated by a maximum associated with the thermodynamically unstable $\mathrm{G}_{1}$ branch. Convexity of $W(Q)$ is restored by the usual Maxwell common-tangent construction. The slope of the common tangent determines the order parameters $Q_{L}$ and $Q_{G_{2}}$ of the delocalized (supercooled liquid) and localized (glass) states, as well as the inter-replicas coupling $\varepsilon_{12}$ via eqn. (12):

$$
W^{\prime}\left(\Gamma, Q_{L}\right)=W^{\prime}\left(\Gamma, Q_{G_{2}}\right)=\varepsilon_{12}
$$

The configurational entropy per particle $s_{\text {conf }}$ finally follows from ${ }^{9}$ :

$$
T^{*} s_{\text {conf }}=W\left(\Gamma, Q_{G_{2}}\right)-W\left(\Gamma, Q_{L}\right)
$$

The values of $Q_{L}, Q_{G_{2}}, \varepsilon_{12}$ and $s_{\text {conf }}$ are listed in Table I for several values of $\Gamma$. The scenario 


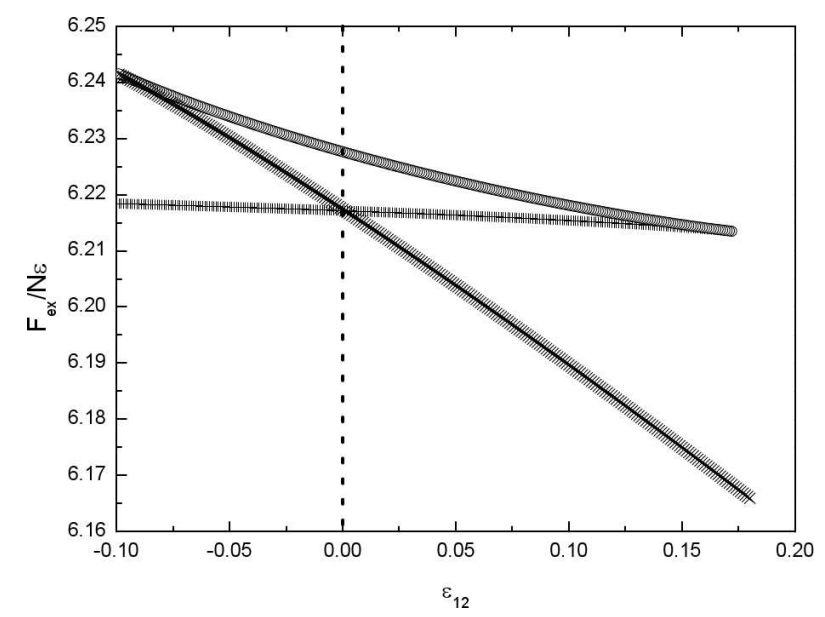

FIG. 6. Dimensionless free energy per particle of the two-replicas system versus $\varepsilon_{12}$ for $\Gamma=1.64$ (HNC and $c / \sigma=0.3$ ). The pluses, circles and crosses are for the $\mathrm{L}, \mathrm{G}_{1}$ and $\mathrm{G}_{2}$ branches respectively.

is similar for $\Gamma=1.63$ and 1.64 as shown in Fig. 5. The key parameters corresponding to a common-tangent construction are listed in Table I.

HNC results for $\Gamma=1.64$, i.e. close to the RFOT, are shown in the next two figures. The excess free energies per particle $F^{e x}\left(\Gamma, \varepsilon_{12}\right) / N \varepsilon_{12}$ are plotted as function of $\varepsilon_{12}$ in Fig.6. Starting from the $\mathrm{L}$ branch at $\varepsilon_{12}=0$, the inter-replicas coupling is gradually increased until the $\mathrm{G}_{1}$ branch is reached at $\varepsilon_{12} \simeq 0.174$. Upon subsequently lowering $\varepsilon_{12}$, the free energy of the $\mathrm{G}_{1}$ branch rises above that of the supercooled liquid. $\varepsilon_{12}$ is extended to negative values (corresponding to a repulsion between atoms of opposite replicas), and at $\varepsilon_{12} \simeq-0.10$, the $\mathrm{G}_{2}$ branch is reached. Upon subsequently increasing $\varepsilon_{12}$, the free energy of the $\mathrm{G}_{2}$ branch drops rapidly below that of the $G_{1}$ branch and crosses the free energy curve of the L branch very close to $\varepsilon_{12}=0$, thus implying that the RFOT to the ideal glass occurs close to $\Gamma=1.64$, in agreement with our earlier prediction ${ }^{14,15}$. The variation of the inter-replicas p.d.f. with $\varepsilon_{12}$ at zero separation, $g_{12}(r=0)$, is shown in Fig. $7 . g_{12}(r=0)$ provides an alternative order parameter to $\mathrm{Q}$ and exhibits a similar, van der Waals-like behavior (cf. Fig.4). The variation of the effective potential $W(Q)$ is similar to that shown in Fig. 5 for lower values of $\Gamma$, and the values of the corresponding parameters, as determined by the common tangent construction are listed in table I. As expected, the configurational entropy $s_{\text {conf }}$ is practically zero, confirming the coincidence of the RFOT critical temperature $T_{o}$, and of the Kauzmann temperature $T_{K}$. A cross-over is expected to occur between the low $\Gamma$ regime (close to $\Gamma_{c}$ ) and the high $\Gamma$ regime (just below $\Gamma_{o}$ ). Fig. 8 compares the HNC 


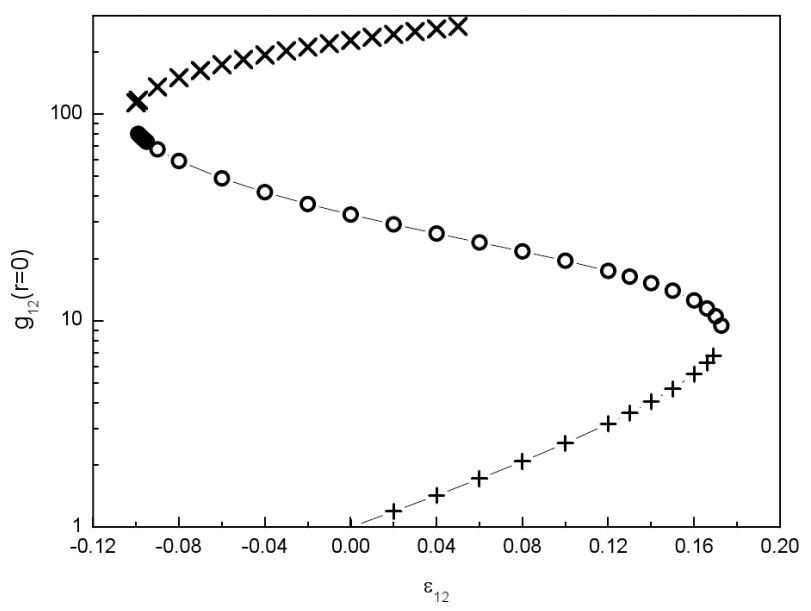

FIG. 7. Zero separation value of the inter-replicas p.d.f. $g_{12}(r=0)$ as a function of $\varepsilon_{12}$, for $\Gamma=$ 1.64; symbols as in Fig. 6 ( $\mathrm{HNC}$ and $c / \sigma=0.3$ ). Note the small discontinuities between $\mathrm{L}$ and $\mathrm{G}_{1}$ branches, and between $\mathrm{G}_{1}$ and $\mathrm{G}_{2}$ branches.

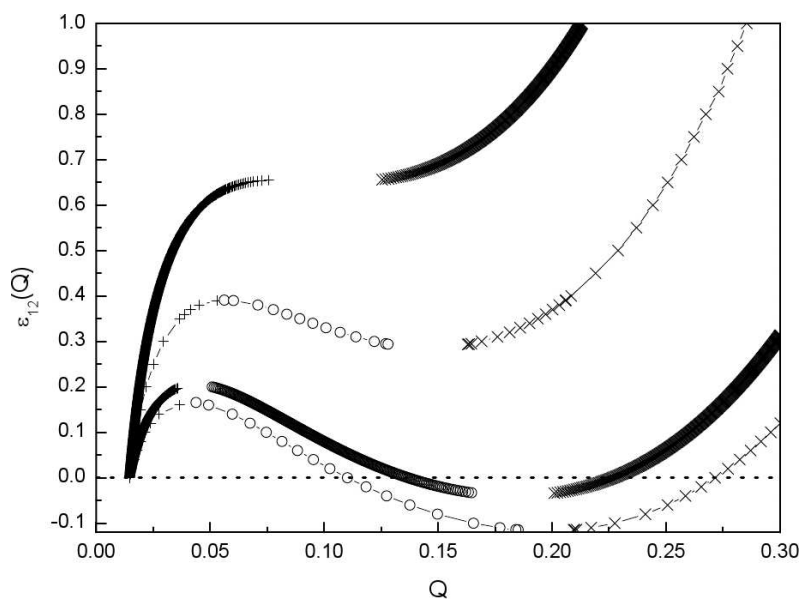

FIG. 8. Same as Fig. 1, but for $\Gamma=1.34,1.45,1.60$, and 1.65 (from top to bottom); symbols as in Fig. $6(\mathrm{HNC}$ and $c / \sigma=0.3)$.

results for $\varepsilon_{12}(Q)$ at an intermediate value, $\Gamma=1.45$, to the data for $\Gamma=1.34$ (cf. Fig. 1) and for $\Gamma=1.6$ (cf. Fig.4) and $\Gamma=1.65$. The pronounced loop observed in the latter case is strongly reduced at $\Gamma=1.45$, and may be expected to disappear in favor of a single discontinuity at a value $1.34<\Gamma \lesssim 1.45$.

The $\varepsilon_{12}$ "phase coexistence" curve is shown in Fig. 9. Beyond the critical coupling $\Gamma_{c} \simeq 1.325$, this curve separates low $\mathrm{Q}$ states (below the coexistence line) from high $\mathrm{Q}$ states (above the line), which may be associated with metastable "supercooled liquid" and 


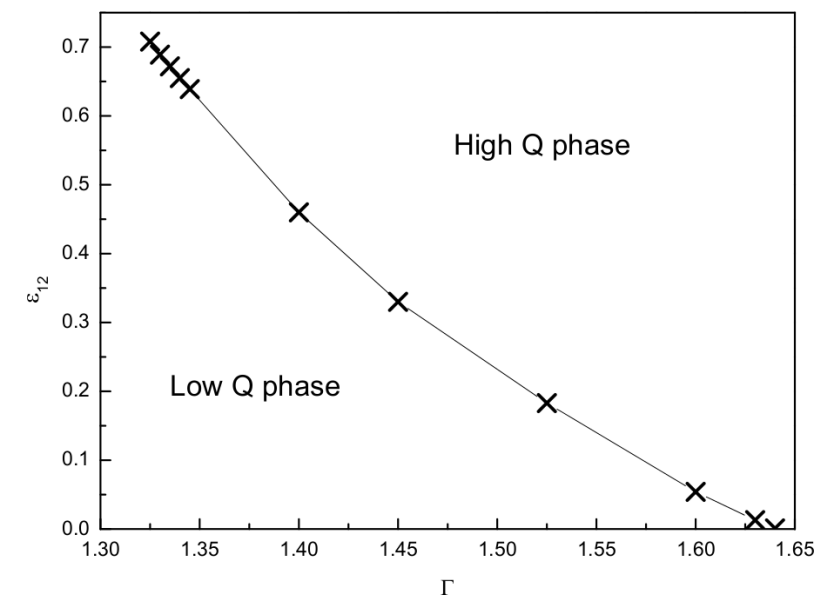

FIG. 9. Coexistence curve of the $\mathrm{L}$ (low Q) and $\mathrm{G}_{2}$ (high Q) phases in the $\left(\varepsilon_{12}, \Gamma\right.$ ) plane (HNC and $c / \sigma=0.3)$.

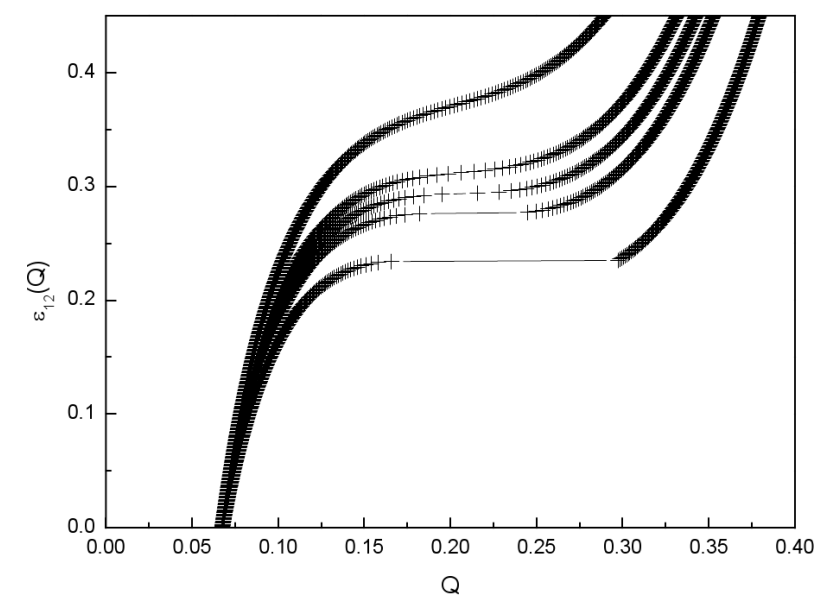

FIG. 10. Same as Fig. 1, but for $c / \sigma=0.5$. From top to bottom: $\Gamma=1.37,1.4,1.41,1.42$ and 1.45 .

glassy phases.

Except in the limit $\varepsilon_{12} \rightarrow 0$, the prediction for the two-replicas system are expected to depend on details of the inter-replicas potential (2), and in particular on the range parameter $c$ in eq. (3). We have briefly investigated the case $c=0.5$ in Fig. 10. The critical coupling $\Gamma_{c}$ above which the order parameter $\mathrm{Q}$ undergoes a discontinuity is estimated to be between $\Gamma_{c}=1.4$ and $\Gamma_{c}=1.42$. The corresponding coupling $\varepsilon_{12} \simeq 0.25$ is significantly lower than its value for $c=0.3$, as may be expected because of the larger range of the attraction between atoms of opposite replicas. However, for values of $\Gamma$ closer to the expected RFOT, the behavior observed with $c=0.5$ is close to than shown in Fig. 4 and 5, as illustrated in 


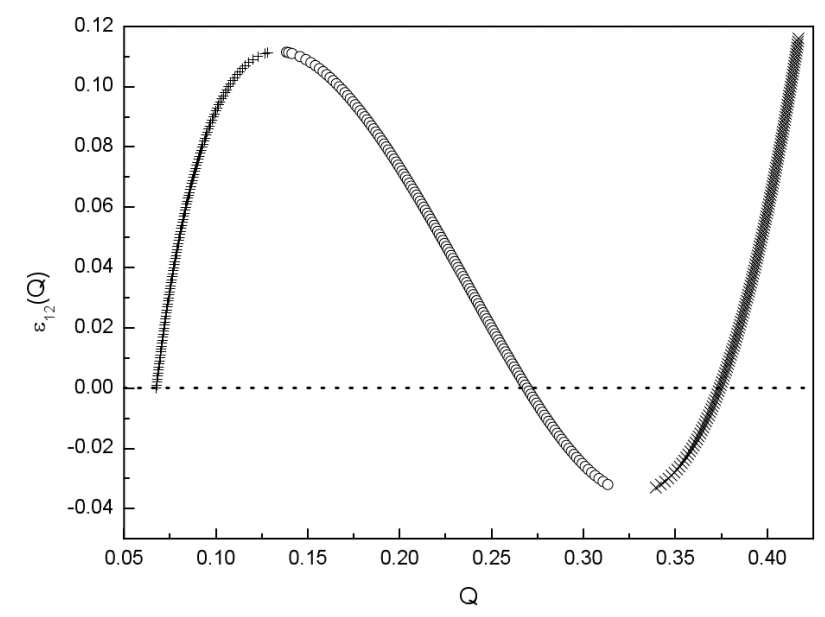

FIG. 11. Same as Fig. 4, but for $c / \sigma=0.5$

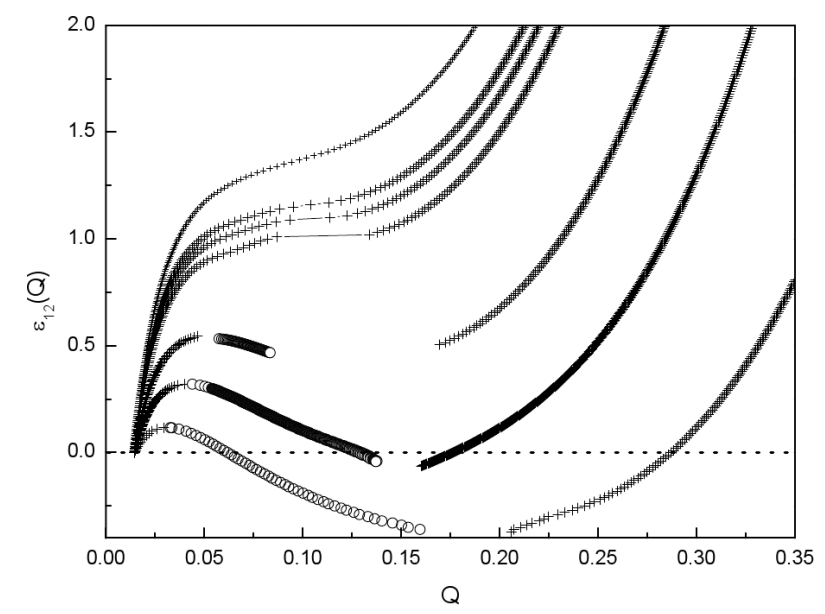

FIG. 12. $\varepsilon_{12}$ versus Q "isotherms" obtained with the RY closure, and $c / \sigma=0.3$. From top to bottom: $\Gamma=1.15,1.18,1.19,1.205,1.3,1.4$ and 1.6.

Fig. 11.

\section{B. RY results}

In order to improve on the HNC results, we now turn to the more accurate RY equation for the calculation of the pair structure of the two-replicas system (cf. Appendix A). In ${ }^{14,15}$ it was shown that the RY equation leads to a similar scenario for RFOT than predicted by HNC, but with significant quantitative differences. In particular, $\Gamma_{o}^{R Y}=1.91$ compared to $\Gamma_{o}^{H N C}=1.65$.

Recently, we detected an error in our code, which affects only previous estimates of the 


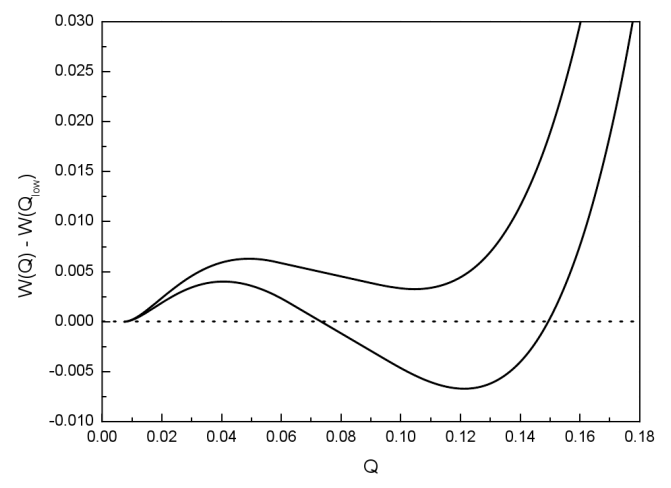

FIG. 13. RY results for the effective potential W(Q) versus $\mathrm{Q}$ at $\Gamma=1.45$ (upper curve) and 1.5 (lower curve) $(c / \sigma=0.3)$; these data should be compared to the corresponding HNC results in Fig. 5.

RY free energy via thermodynamic integration. The corrected value of $\Gamma_{o}^{R Y}$, as obtained from the difference in free energy between the $G_{2}$ and $L$ branches (using $c=0.3$ ) is 1.66, remarkably close to the HNC prediction. Interestingly, although the enforcement of the virial-compressibility consistency greatly improves the overall quality of structural and thermodynamic RY results, it introduces a path dependence in the thermodynamic integration, which in turn induces an inconsistency between $\Gamma_{o}^{R Y}$ obtained from the free energy difference, and from $W\left(Q_{G 2}\right)-W\left(Q_{L}\right)$. The RY results for $\varepsilon_{12}(Q)$ are shown in Fig. 12. The qualitative behavior is reminiscent of the HNC results shown in Fig. 1, including the presence of small intervals where no solution can be found, but the critical coupling is estimated to be $\Gamma_{o}^{R Y} \simeq 1.20$, well below the value $\Gamma_{c}^{H N C} \simeq 1.325$; the corresponding values of $\varepsilon_{12}$ also differ substantially ( $\varepsilon_{12} \simeq 1.0$ compared to $\varepsilon_{12} \simeq 0.68$ ). The appearance of two additional $Q$ values at which the slope of $\varepsilon_{12}(Q)$ vanishes, occurs at a lower coupling. We observe that the appearance of the precursor $\left(\mathrm{G}_{1}\right)$ branch (the part of the curves where $\varepsilon_{12}(Q)$ decreases as $Q$ increases) is also confirmed at the RY level. However, solving the RY integral equation along this branch is not an easy task: to the left of the minimum where the $G_{2}$ branch starts, an interval of $Q$ values appears where the reason for absence of solution is the impossibility of achieving thermodynamic consistency between virial and compressibility equations of state. It would be instructive to check the quality of alternative advanced closure relations along the $\mathrm{G}_{1}$ branch. 
The corresponding $W(Q)$ curves are shown in Fig. 13. The resulting estimate of $\Gamma_{o}^{R Y}$ is close to 1.47 , which is significantly lower than the estimate $\Gamma_{o}^{R Y}=1.66$ quoted above, as obtained from the free energy difference between $L$ and $G_{2}$ branches. This difference quantifies the residual inconsistency of the RY closure.

\section{DISCUSSION AND CONCLUSIONS}

Our results for the overlap order parameter and effective potential of a system of two weakly coupled replicas of a soft sphere model, based on careful numerical solutions of the HNC and RY integral equation over a wide range of the inter-replicas coupling $\varepsilon_{12}$, confirm the existence of a "precursor transition" between high and low overlap phases above the ideal glass transition temperature associated with the limit $\varepsilon_{12} \rightarrow 0$. Such a transition was predicted in a number of recent publications ${ }^{8-10,12,13}$, on the basis either of related integral equation approaches or of MC simulations. The results presented in this paper are restricted to an "annealed" two replicas system, as opposed to the "quenched" case, where the free energy of a system is first calculated in the external field of a frozen disordered configuration of a similar system, and a statistical average over the disorder is taken at the end.

Some significant differences between our predictions and earlier work emerge however. The main difference lies in the appearance of an additional glass branch $\mathrm{G}_{1}$, intermediate between the $\mathrm{L}$ and $\mathrm{G}_{2}$ (ideal glass) branches, which results in a characteristic S-shaped loop in the $\varepsilon_{12}(Q)$ curves below a cross-over temperature $T_{c}>T \geq T_{o}\left(\right.$ or $\left.\Gamma_{c}<\Gamma \leq \Gamma_{o}\right)$.

The presence of the $G_{1}$ branch in integral equation calculations should be seen as an important confirmation of the "precursor transition" scenario. The putative $\mathrm{G}_{1}$ branch appears in our numerical solutions of both HNC and RY integral equations, and is also observed for a two replicas Lennard-Jones system ${ }^{16}$, but was not detected in related work by other groups. Its existence allows a near-continuous path between the physically relevant L and $\mathrm{G}_{2}$ branches, but does not affect the characteristic two-minima structure of the effective potential $W(Q)$ associated with low $\mathrm{Q}$ and high $\mathrm{Q}$ states below $T_{c}$. The $G_{1}$ branch is eliminated by a Maxwell construction, required for restoring the proper convexity of the equilibrium free energy.

Thus, the structural robustness of the existence of a precursor transition predicted theoretically in the literature $\mathrm{e}^{8,9,12,13}$ is confirmed by the present work, and in particular by the 
similarity of the scenarii predicted by the HNC and RY calculations even if the latter imply significantly different values of $T_{c}$. Although the existence of the transition driven by pairing of atoms of opposite replicas to form a "molecular phase", is not in itself surprising from a theoretical point of view, one may wonder about its physical relevance and observational implications. Several suggestions have been put forward concerning possible links of the precursor transition with atomic dynamics in deeply supercooled liquids, including dynamical heterogeneities (see e.g. ${ }^{23,24}$ ) but further investigations are required to link the transition to purely static properties.

\section{APPENDIX A}

The pair structure of a symmetric binary mixture is characterized by two pair correlation functions, $h_{11}(x)=g_{11}(x)-1=h_{22}(x)$, and $h_{12}(x)=g_{12}(x)-1$, or, alternatively, by two direct correlation functions $c_{11}(x)=c_{22}$ and $c_{12}(x)$; the two sets are linked by two coupled Ornstein-Zernike $(\mathrm{OZ})$ relations ${ }^{17}$ :

$$
h_{\mu \nu}(x)=c_{\mu \nu}(x)+\rho^{*} \sum_{\lambda=1,2} c_{\mu \lambda}(x) \otimes h_{\lambda \nu}(x) ; 1 \leq \mu, \nu \leq 2
$$

where $\otimes$ denotes a convolution product. The OZ relations must be supplemented by approximate closure relations; defining $\gamma_{\mu \nu}(x)=h_{\mu \nu}(x)-c_{\mu \nu}(x)$, we have adopted the HNC and RY closures:

$$
\begin{array}{r}
g_{\mu \nu}(x)=\exp \left\{-\beta v_{\mu \nu}(x)+\gamma_{\mu \nu}(x)\right\} \quad(H N C), \\
g_{\mu \nu}(x)=\exp \left\{-\beta v_{\mu \nu}(x)\right\}\left[1+\frac{\exp \left\{f_{\mu \nu}(x) \gamma_{\mu \nu}(x)-1\right\}}{f_{\mu \nu}(x)}\right]
\end{array}
$$

In the RY closure ${ }^{18}$, the $f_{\mu \nu}(x)=1-\exp \left\{-\alpha_{\mu \nu}(x)\right\}$ are switching functions which depend on the inverse range parameters $\alpha_{11}=\alpha_{22}$ and $\alpha_{12}$; for the latter we take the limit $\alpha_{12} \rightarrow \infty$, (i.e. $f_{12}(x)=1$ ), while $\alpha_{11}$ is varied to achieve thermodynamic self-consistency between virial and long-wavelength compressibility. Note that in the limit $\alpha_{11} \rightarrow \infty$, the RY closure reduces completely to the HNC closure (20a). The coupled integral-equations resulting from the combination of the OZ relations (19) and the closure relations(20a) and (20b) were solved numerically using Gillan's method ${ }^{25,26}$ over a wide range of state variables $\Gamma$ and $\varepsilon_{12}$, with a real space grid of $\Delta x=0.02$, and $2^{12}$ or $2^{13}$ intervals, ensuring that the pair correlation functions have decayed to values of the order od $10^{-12}$ or less. The order parameter $Q\left(\Gamma ; \varepsilon_{12}\right)$ 
is calculated from eqn. (5); the $g_{\mu \nu}(x)$ also allow the calculation of the excess internal energy and the equation of state via the standard energy, virial and compressibility equations ${ }^{17}$. Within HNC, the excess free energy is also directly accessible by the pair structure via the HNC expression for the excess chemical potential ${ }^{14,15}$ :

$$
\beta \mu_{e x}\left(\Gamma ; \varepsilon_{12}\right)=\frac{\rho^{*}}{2} \sum_{\nu=1,2} \int\left[h_{1 \nu}(x) \gamma_{1 \nu}(x)-2 c_{1 \nu}(x)\right] d \mathbf{x}
$$

This relation does not hold within the RY approximation, and hence the excess free energy must be calculated by thermodynamic integration of the excess internal energy if one does not wish to introduce additional approximations. The calculations are straightforward for the supercooled liquid(L) branch which extends down to $\Gamma=0$. Since the glass branches $G_{1}$ and $\mathrm{G}_{2}$ terminate below a threshold coupling $\Gamma_{0}$, more complicated thermodynamic paths (or "protocols") must be used, which are detailed elsewhere ${ }^{14,15}$. The effective potential $W(\Gamma ; Q)$ finally follows from eqs. 5 and 10.

\section{ACKNOWLEDGMENTS}

The authors would like to thank P. Charbonneau, L. Berthier, F. Zamponi, S. Franz and B. Seoane for useful discussions.

\section{REFERENCES}

${ }^{1}$ W. Götze, Complex Dynamics of Glass-Forming Liquids: a Mode-Coupling Theory (Oxford University Press, New York, 2009).

${ }^{2}$ H. Sillescu, Journal of Non-Crystalline Solids 243, 81 (1999).

${ }^{3}$ M. Ediger, Annual review of physical chemistry 51, 99 (2000).

${ }^{4}$ P. G. Debenedetti and F. H. Stillinger, Nature 410, 259 (2001).

${ }^{5}$ P. Charbonneau, J. Kurchan, G. Parisi, P. Urbani, and F. Zamponi, Nature Communications 5, 4725 (2014).

${ }^{6}$ A. W. Kauzmann, Chemical Reviews 43, 219 (1948).

${ }^{7}$ L. Berthier and G. Biroli, Reviews of Modern Physics 83, 587 (2011).

${ }^{8}$ S. Franz and G. Parisi, Physica A 261, 317 (1998).

${ }^{9}$ M. Cardenas, S. Franz, and G. Parisi., The Journal of chemical physics 110, 1726 (1999). 
${ }^{10}$ L. Berthier and R. L. Jack, Physical Review E 76, 041509 (2015).

${ }^{11}$ J. A. Given and G. R. Stell, Physica A 209, 495 (1994).

${ }^{12}$ L. Berthier, Physical Review E 88, 0222313 (2013).

${ }^{13}$ G. Parisi and B. Seoane, Physical Review E 89, 022309 (2014).

${ }^{14}$ J.-M. Bomont, J. P. Hansen, and G. Pastore, Journal of Chemical Physics 141, 174505 (2014).

${ }^{15}$ J.-M. Bomont, J. P. Hansen, and G. Pastore, Journal of Chemical Physics 142, 107105 (2015).

${ }^{16}$ J.-M. Bomont, J. P. Hansen, and G. Pastore, Physical Review E 92, 042316 (2015).

${ }^{17}$ J. P. Hansen and I. R. McDonald, Theory of Simple Liquids, 4th ed. (Elsevier, Amsterdam, 2013).

${ }^{18}$ F. J. Rogers and D. A. Young, Physical Review A 30, 999 (1984).

${ }^{19}$ G. Zerah and J. P. Hansen, Journal of Chemical Physics 84, 2336 (1986).

${ }^{20}$ M. Mézard and G. Parisi, Journal of Chemical Physics 111, 1076 (1999).

${ }^{21}$ L. Belloni, Journal of Chemical Physics 98, 8080 (1993).

${ }^{22}$ E. Lomba and J. López-Martín, Journal of Statistical Physics 80, 825 (1995).

${ }^{23}$ C. Cammarota, A. Cavagna, I. Giardina, G. Gradenigo, T. S. Grigera, and G. Parisi, Physical Review Letters 105, 055703 (2010).

${ }^{24}$ A. Ninarello, L. Berthier, and D. Coslovich, Molecular Physics 113, 2707 (2015).

${ }^{25}$ M. J. Gillan, Molecular Physics 38, 1781 (1979).

${ }^{26}$ G. M. Abernethy and M. J. Gillan, Molecular Physics 39, 839 (1980). 